\title{
Exploiting Knowledge Based Systems to Support Manufacturing of Functional Food Products
}

\author{
Stefania Bandini ${ }^{2}$, David Martínez-Simarro ${ }^{1}$, J.M. Pinazo Sánchez ${ }^{1}$, \\ Fabio Sartori ${ }^{2}$, and Giuseppe Vizzari ${ }^{2}$ \\ ${ }^{1}$ Ainia centro tecnológico Valencia-Spain \\ ${ }^{2}$ CSAI (Complex Systems and Artificial Intelligence research centre-University of \\ Milano-Bicocca)
}

\begin{abstract}
In recent years consumers' concern about food safety and health is becoming crucial. The development of healthier products seems to be a promise challenge, since functional foods are identified as one of the central pillars for the future progress of the food industry internationally. Artificial Intelligence techniques such as Knowledge Engineering, Rule Based reasoning and Expert systems are presented here as a way of technological innovation towards a knowledge-based and sustainable manufacturing in food sector. We show how these techniques are applied in a case study to support companies in the formulation of new chocolate products that reduce the impact on health status, reducing selected risk factors associated with obesity and coronary disease (fat, sugar).
\end{abstract}

\section{Introduction}

In last years the interest of technology platforms ${ }^{1}$, public health organizations and consumers in knowing the relation between diet and health has increased considerably. The experts in nutrition recommend to follow a healthy, diverse and balanced diet as the best way of preventing or at least reducing the risk of suffering certain alterations or diseases in short and long term: hypertension, obesity, diabetes, cardiovascular diseases, disorders on food habits and even certain types of cancer related to the nourishment.

Consumers are increasingly interested in the health benefits of foods and have started to look beyond the basic nutritional benefits of food to the disease prevention and health enhancing compounds contained in many foods. This combined with a more widespread understanding of how diet affects disease, health-care costs and aging populations have created a potentially sustainable market for functional foods.

The aim of this paper is to propose an approach based on the exploitation of knowledge artifacts [1] in a collaborative environment to support the development of functional products. In this paper we present a conceptual and computational framework for the development of knowledge-based systems (KBS) to support experts of small and medium enterprises involved in the formulation of functional healthier food products. The paper investigates how functional knowledge can be profitably acquired

\footnotetext{
${ }^{1}$ European Technology Platform Food For Life, http://etp.ciaa.be
} 
and represented through the usage of a specific knowledge artifact, namely ontologies and T-Matrix [2]. Ontologies [3] are one of the most suitable approaches to represent functional knowledge about a domain. In our framework, ontologies are used to specify the structure of the food product (formula) with respect to the functional nature of the relationships existing among its raw material or additives (ingredients). The $\mathrm{T}$ Matrix tool allows representing how different structural ingredients of a food product can be aggregated from the functional point of view (nutritional properties), as well as the relationships existing between functions and the final performances (performance properties) the product must have to be successful. T-Matrix is thus a specific model for the representation of such ontological aspects. The participation of a KBS in this case guarantees both the sharing of the knowledge model among users (user defined ontology) and suggesting solutions to the compounding problem [1] (rule based decision support). The proof of concept we have realised enables an intuitive and highly integrated formula and recipe development, collaboration tools and global regulatory compliance support.

The related case study shows an experiment of our intended application to the reformulation of chocolate products. The work was related to modify the ingredient profile of the standard recipe of traditional products, increasing some ingredients while reducing others in conjunction with maintaining commercial viability.

This paper is organized as follows: The next Section 2 deals with the context and motivation of the related work. In Section 3, a flexible and extensible approach to the support of formulating new food products based on knowledge systems is presented. The design strategy, the domain vocabulary and discussion on the proposed approach are offered as well. Later on, a case study is presented there by means of the application of the approach to a specific problem: the reformulation of chocolate products. Finally, Section 4 points out some remarks and future works.

\section{Context and Motivation}

A functional food ${ }^{2}$ is one that has demonstrated a significant effect on the body beyond adequate nutrition, in a way that improves health and well-being and reduces disease. Functional foods provide an opportunity to improve the health of European inhabitants and reduce health care costs while support economic development in several areas. Researchers, scientific community and food industry work all together with the aim to promote the concept of healthy food. Recent advances in molecular biology and nutrition technologies give food industry a starting point for the design and development of new ingredients and additives for healthy life styles [4-5].

In order to achieve healthier food products and derivatives (even with functional properties), it is necessary to avoid undesired substances (natural or otherwise) or reduce them to appropriate limits, and to increase the levels (naturally or by programmed additions) of other substances with beneficial properties (functional ingredients). Essentially, three kinds of strategies are used to that end in food sector: these are associated with animal or plant production, the handling and processing of raw materials, and the reformulation of intermediate or final products.

\footnotetext{
${ }^{2}$ European Food Information Council (EUFIC). http://www.eufic.org
} 
At this stage reformulation is used as far as possible to develop a range of derivatives with custom-designed composition and properties. To that purpose, there are two possible types of complementary intervention. The first involves reducing some compounds normally present in these foods to appropriate amounts, for example, fat, Saturated Fatty Acids (SFA), salt, sugar, nitrites and so on. The second is to incorporate ingredients that are potentially health-enhancing (functional), for example, fibre, certain types of vegetable proteins, Mono Unsaturated Fatty Acids (MUFA) and Poly Unsaturated Fatty Acids (PUFA), antioxidants, etc.

There are numerous aspects to consider in the development of this kind of products [6]. The new derivative product must have the appropriate technological, sensory and nutritional properties, and be safe and convenient for consumption. Ignoring such requirements, which are demanded by the reference products if they are to be improved, not only compromises the success of the derivatives concerned but also projects a bad product image and creates a lack of confidence which is difficult to surmount.

In the reformulation process, compounding problem stands for the research of the most suitable way of combining ingredients in order to design a product described by a recipe [1]. This indicates which ingredients are involved in the construction of the product by the indication of their amount. The problem has been tackled involving the use of KBS in several domains. For instance the compounding problem has been faced in tablets [7] and colors [8] design and, in particular, in rubber compounding [9].

In the food domain, several new products are launched to the market every year ${ }^{3}$. When companies try to reformulate, the typical situation is that a technically skilled expert has specific knowledge about the domain (ingredients, processes and products). Therefore, the master has the adequate expertise to combine relevant entities in an appropriate way to achieve the desired goal: the formulation of a new product with functional benefits. Not surprisingly, this approach is totally valid, but modern information technologies could be thoroughly integrated into everyday activity of food product formulation and improve the efficiency and effectiveness of related tasks:

First of all, thanks to the use of knowledge elicitation techniques and representation, the development of new products could be extended beyond the basic formulation of a recipe to include marketing considerations. By this way, product innovation is not only aligned to the basic specifications and regulatory compliance but also to the product nutritional properties, performance needs and consumer preferences and acceptances, such as price, format (objective), as well as taste, noise, color, flavour, texture and so on (subjective). See Figure 1 below with the aimed approach.

Secondly, to revise a recipe and assess the development of healthier products, a KBS can be used to support decision making processes [10] in the formulation of safety derivatives both of skilled and expert users and of beginners. The participation of a KBS in this process is threefold:

- sharing the knowledge model (i.e. the contents of the knowledge base) among involved users,

- training beginner/non-expert users (i.e. allowing a rapid technology transfer and prototyping: virtual lab)

- and suggesting solutions to particular problems (i.e. the results of computations made by the inferential engine on the contents of the knowledge base).

\footnotetext{
${ }^{3}$ Global National Product Database, http://www.gnpd.com
} 


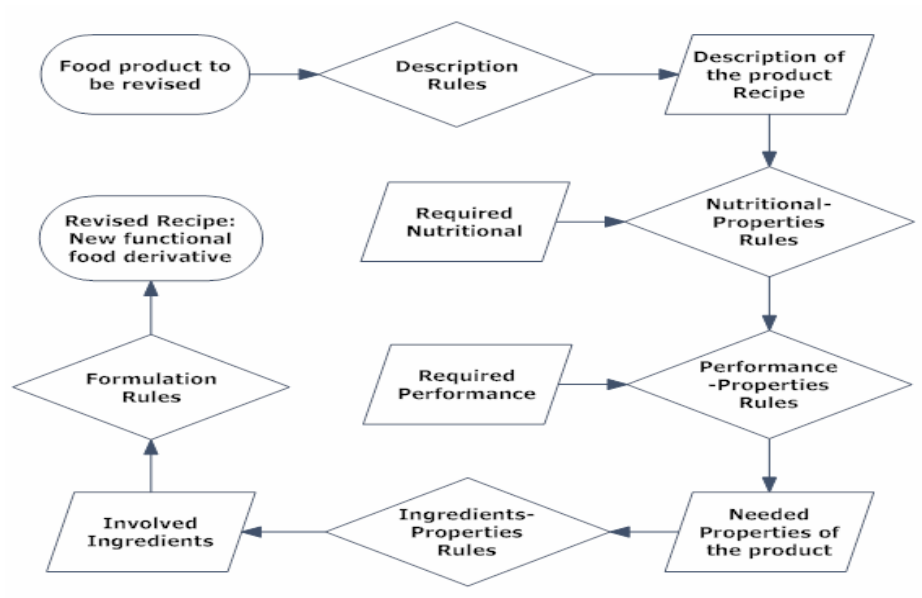

Fig. 1. Challenging approach for functional food formulation

The effectiveness of the KBS and the quality of its contribution in supporting decision making processes will depend mainly on two classes of aspects: internal (e.g. correctness and integrity) and external (e.g. adherence with the application domain and maintainability level).

In our aforementioned approach we apply the KBS to:

- The resolution of a real problem by means of the use of knowledge from the application domain.

- The resolution of a problem that requires a great amount of expert knowledge when it is solved by not experts or beginners.

Not in a substitution approach, but rather with the aim of smoothing the decision making process.

\section{Application Scenario}

The chocolate and other derivatives of the cocoa have been consumed in diverse forms throughout centuries. Nowadays, chocolates can be found in several ways and formats: tablets, pills, liquid chocolate, sweet, nougats, creams, etc. Basic ingredients of its composition are the cocoa solids and cocoa butter, fats and oils (whose composition obey rheological characteristics and about melting point adapted to every need), sugar, milk powder and / or lacteal and dried fruits (almond, hazelnut, nut ...). All that does chocolate a particularly energetic product for the presence of glucides and lipids: from 500 to $550 \mathrm{Kcal}$ for $100 \mathrm{~g}$. The half of this contribution comes from the present lipids in several raw materials. The oily total contribution in chocolates registers from 25 to $40 \%$ of the weight of the product. This proportion difficultly can diminish, since it is requested by production needs or by the organoleptic perception for the final consumer. 


\subsection{Chocolate Recipe Characterization}

Within this context, the tackled problem is the formulation of components in new food product development. The results of compounding activity are fundamental for the definition of the raw materials and additives (i.e. the ingredients) to be used in the formulation of the product. The amount of each ingredient is measured in \% values. In food domain, nutritional properties are of paramount importance since they represent our needs for vitamins, minerals, fatty acids and proteins. For instance, the consumption of too many saturated fats is the leading cause of high cholesterol. Unsaturated fats are healthier in moderation than saturated fats. Proteins are essential nutrients for growth and digestion.

The first objective of the project has been a complete characterization of food product from the structural (nutritional properties) and functional (performance) point of view. To this aim, an ontology of the product (chocolate) has been defined and implemented in Protégé. The hierarchical organization of the different concepts and individuals of the ontology is partially represented in Figure 2.

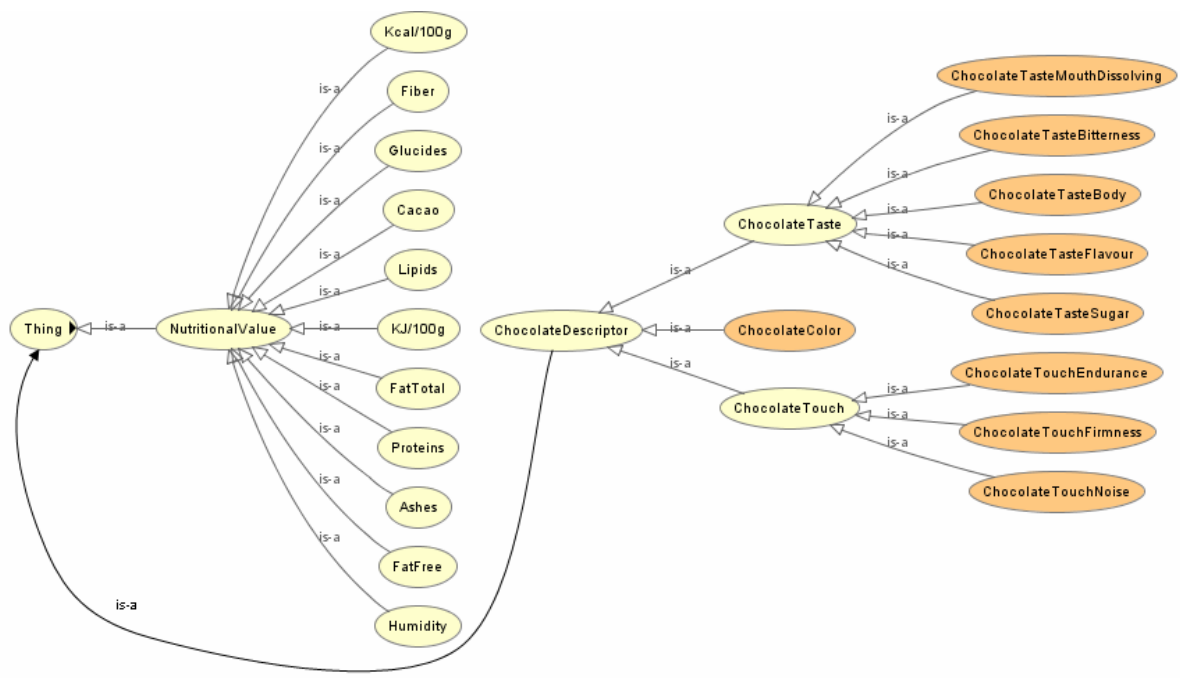

Fig. 2. Partial representation of chocolate ontology

Domain entities resulting from the performed ontological analysis are distributed in recipe names, ingredients, nutritional and performance properties. These constitute the Terms of the grammar.

$<$ nutritional property> ::= "np1" | "np2" | ...

$<$ performance property $>::=$ "pp1" | "pp2" | ...

$<$ recipe $>::=$ "RECIPE_1" | "RECIPE_2" | ...

$<$ ingredient> ::= "ingredient_1" | "ingredient_2" | ... 
A previously reported, recipes are made up of ingredients, each one belonging to a family (e.g. sugars, lacteals, flours).

$$
<\text { family }>::=\text { "F1" | "F2" | "F3" | ... }
$$

For instance, the recipe name Black Chocolate involves ingredients Cacao Butter, Cacao Powder, sugar, vanilla and lecithin. Each ingredient has a nutritional property table that implies a side effect in the final recipe.

Within a recipe it is possible to identify sets of ingredients made up of elements of the same family and with a role in the final formula. Each role is a functional group of ingredients (system), inserted in the recipe in order to provide particular feature to the final recipe (e.g. basis, sweeteners, flavourings).

$$
\text { <system > ::= "S1" | "S2" | "S3" | ... }
$$

By this way, it is possible to represent if there is an association between each ingredient (i.e. cacao powder) and its influence in the final recipe as in Figure 3.

Some constraints that define boundaries for the construction of recipes suitable for derivative development can be described. Generally, these constraints depend on the purpose of the product. It is the case of constraints on combinations cardinality, combination amount, ingredient amounts and continuous attribute value limits.

These constraints (description, nutritional, performance, ingredients and formulation rules) will be defined with the help of a rule engine. But, initially, we make use of a T-Matrix (see Table 1) to represent the correlations among ingredients, nutritional properties and performance.

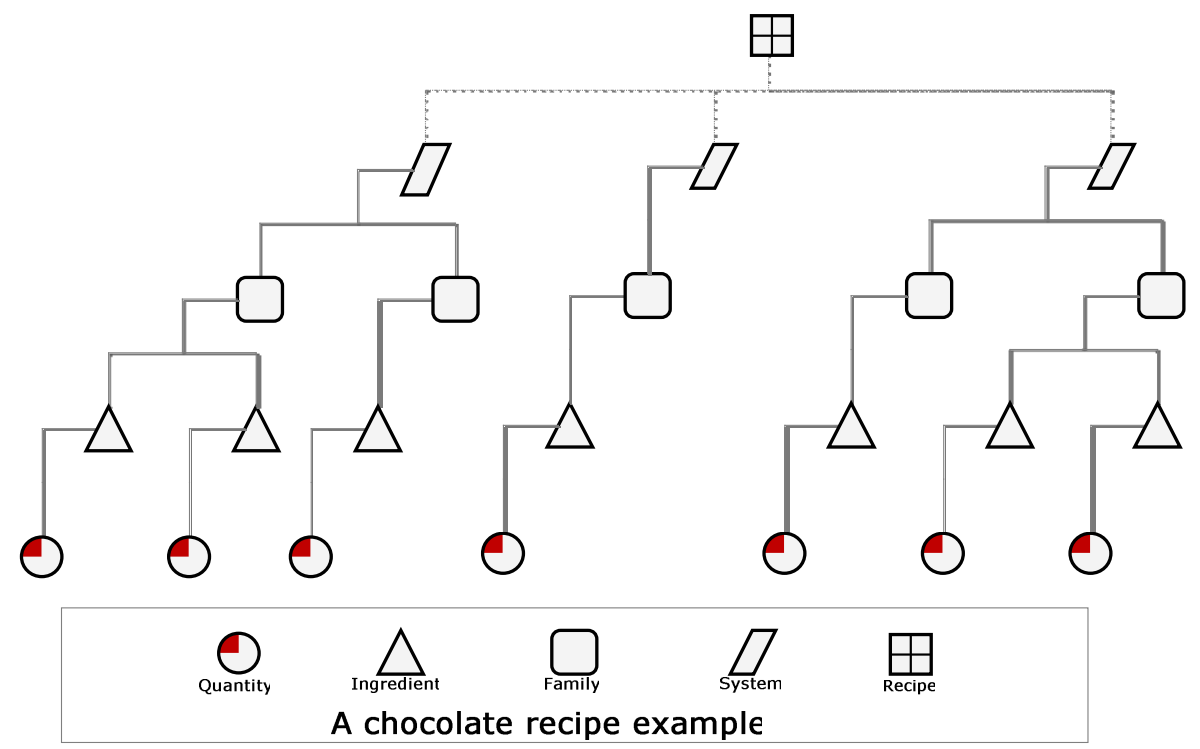

Fig. 3. A chocolate recipe example 
Table 1. T-Matrix for nutritional and high level properties

\begin{tabular}{|l|l|l|l|l|l|l|l|l|}
\hline & Fat & Proteins & Fiber & $\begin{array}{l}\text { Carbo- } \\
\text { hydrates }\end{array}$ & Humidity & Calories & Minerals & Vitamins \\
\hline RI1 & $\uparrow$ & $\times$ & $\times$ & $\times$ & $\times$ & $\uparrow \bullet$ & $\uparrow \square$ & $\uparrow \square$ \\
\hline RI2 & $\uparrow \square$ & $\uparrow \square$ & $\uparrow \bullet$ & $\uparrow \bullet$ & $\uparrow \bullet$ & $\uparrow \square$ & $\uparrow \bullet$ & $\uparrow \bullet$ \\
\hline RI3 & $\times$ & $\times$ & $\times$ & $\uparrow$ & $\times$ & $\uparrow$ & $\downarrow \bullet$ & $\downarrow \bullet$ \\
\hline RI4 & $\times$ & $\uparrow$ & $\times$ & $\uparrow \square$ & $\uparrow \square$ & $\uparrow$ & $\uparrow \square$ & $\uparrow \square$ \\
\hline
\end{tabular}

\begin{tabular}{|c|c|c|c|c|c|c|c|c|}
\hline & Solubility & Bitternesss & Cacao & Cost & Flavour & Color & Texture & Noise \\
\hline RI1 & $\uparrow \square$ & $\downarrow \square$ & $\uparrow \square$ & $\uparrow \square$ & $\uparrow=$ & $\uparrow=$ & $\uparrow \square$ & $\uparrow \square$ \\
\hline RI2 & $\downarrow \square$ & $\uparrow \square$ & $\uparrow \square$ & $\uparrow \square$ & $\uparrow \square$ & $\uparrow \square$ & & \\
\hline RI3 & & $\downarrow \square$ & & & & & & \\
\hline
\end{tabular}

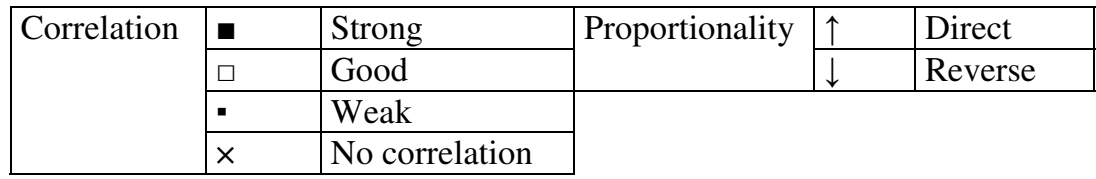

It is possible to create a T-Matrix for each possible formula recipe. For a particular usage of the ingredients the relationships reported in the T-Matrix show the effects of recipe interventions in terms of variations of nutritional properties (i.e. calories) and the effects on product performance (i.e. taste). Two sets of additional terms are respectively dedicated to represent the grade of correlation and the proportionality.

\subsection{Software Prototype}

The proof of concept of the system consists of a knowledge base, a database, and a user interface. The basic architecture of the system is described and two important issues, knowledge representation and natural language processing, are discussed. The aim of the system is to provide the process expert a tool to simplify the recipe transformation process and to help them to control the recipe management activity during the formulation of functional products with health benefits. Knowledge elicitation and representation techniques, and rule based programming combined with object programming (Java + Drools $\left.{ }^{4}\right)$ are employed in the system.

The user interacts with the system through a user interface which may use menus, natural language or any other style of interaction. Then an inference engine is used to reason with both the expert knowledge (extracted from our friendly expert) and data specific to the particular problem being solved. The expert knowledge will typically be in the form of a set of IF-THEN rules. The case specific data includes both data provided by the user and partial conclusions (along with certainty measures) based on

\footnotetext{
${ }^{4}$ Drools Project, http://www.jboss.org/drools/
} 


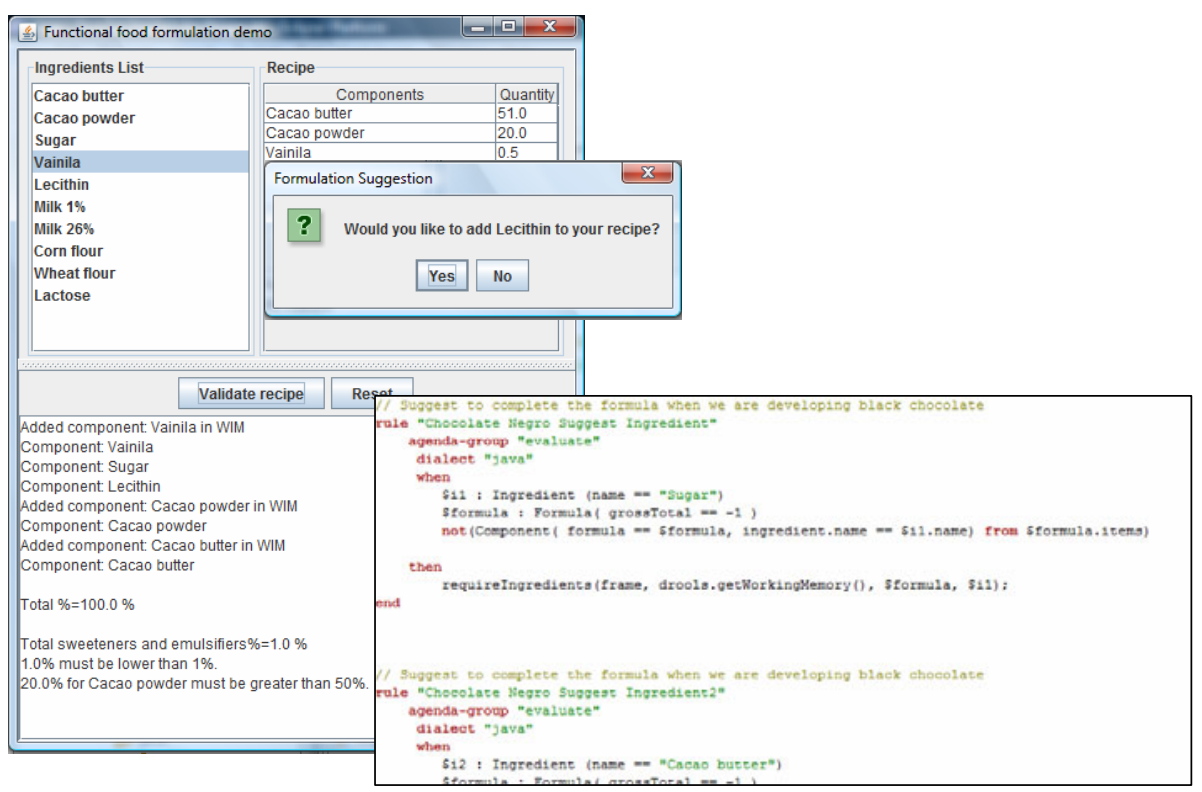

Fig. 4. KBS proof of concept for formulation of functional chocolate

this data. In a simple forward chaining rule-based system the case specific data will be the elements in working memory.

The Figure 4 below illustrates the aspect of the prototype system and shows an intended trial for the modification of the ingredient profile of the standard black chocolate recipe. This included increasing fibre while reducing both fat and sugar.

\section{Concluding Remarks and Future Development}

This work shows an innovative sustainable and knowledge-based product and manufacturing model to support the development of functional food. The present approach looks promising since it allows redefining the way in which food companies' valuecreation process must be conceived and realized, in this case applied to a concrete food scenario for:

- Designing new formulations that include the new potentially interesting ingredients for healthy effects in the targeted product.

- Receiving an assessment and decision support for the generation of new formulations with low content in harmful ingredients (sugar, fat).

- Studying and representing the influence of the different ingredients and additives in the final formula and their relation to performance properties such as color, flavour, taste, smell, image, etc.

Of course our work is a first experiment and the primary future direction is the realisation of a concrete application in collaboration with an end-user. Likewise, due to the 
relevance of the field in the food sector, a challenging line of work is open and future developments could exploit further potentialities of the system:

- Study and represent the influence of the different stages of the process (mixed, warming, etc.) in the selected ingredients and the formula.

- Exploit the ability to reformulate products while keeping the same taste, texture and consumer satisfaction, enabling the interaction between the consumer and the product-life cycle development through a co-innovation network.

- Enrich the access to a wide variety of functional ingredients by means of the integration of several data sources through information retrieval techniques.

- Take the advantage of registered data (physical measures of process, product and environment, actions and other incidents) to automatically generate and retune recipes. On line comparison of product evolution with previous (finished, classified and registered) ones and also after consumers' perception trials could be used to improve productions in terms of quality, productivity or homogeneity. Case Based Reasoning (CBR), could be proposed in order to reach this goal: compounding is performed designing a recipe by adapting a previous one.

- A mixed approach combining RBR and CBR could be also potentially exploited to support product formulation for food safety.

\section{Acknowledgments}

This paper describes some of the results achieved during the collaboration between CSAI in Milano and ainia technological center in Valencia thanks to the "Programa de Alta Especialización de Tecnólogos" financed by the Valencian Institute for SMEs IMPIVA.

\section{References}

1. Bandini, S., Colombo, E., Vizzari, G.: The The Role of Knowledge Artifacts in Knowledge Maintenance, Department of Informatics, Systems and Communication, University of Milano-Bicocca. Via Bicocca degli Arcimboldi, 8, 20126 Milan, Italy (2005)

2. Bandini, S., Sartori, F.: From From handicraft prototypes to limited serial productions: Exploiting knowledge artifacts to support the industrial design of high quality products. In: AIE, Artificial Intelligence for Engineering Design, Analysis and Manufacturing, CSAIComplex Systems and Artificial Intelligence Research Center. University of Milano, Milan (2009)

3. Gruber, T.R.: Toward Principles for the Design of Ontologies Used for Knowledge Sharing. Stanford Knowledge Systems Laboratory 701 Welch Road, Building C Palo Alto, CA 94304, http: / / gruberksl.stanford.edu

4. Pszczola, D.E.: Addressing functional problems in fortified foods. Food Technology 52(7), 38-46 (2001)

5. Pszczola, D.E.: Ingredients that get to meat to matter. Food Technology 53(4), 62-74 (1999)

6. Jiménez-Colmenero: Fat replacers in meat products (2000) 
7. Craw, S., Wiratunga, N., Rowe, R.: Case Based Design for Tablet Formulation. In: Proceedings of the 4th European Workshop on Case Based Reasoning, pp. 358-369. Springer, Berlin (1998)

8. Cheetham, W., Graf, J.: Case-Based Reasoning in Color Matching. In: Leake, D.B., Plaza, E. (eds.) ICCBR 1997. LNCS, vol. 1266, pp. 1-12. Springer, Heidelberg (1997)

9. Bandini, S., Manzoni, S.: Modeling Core Knowledge and Practices in a Computational Approach to Innovation Process. In: Magnani, L., Nersessian, N. (eds.) Model-Based Reasoning: Scientific Discovery, Technologicy, Values, pp. 369-390. Kluwer Academic/Plenum Publishers (2002)

10. Holsapple, C., Joshi, K.: Organizational Knowledge Resources. Decision Support Systems, pp. 39-54 (2001) 